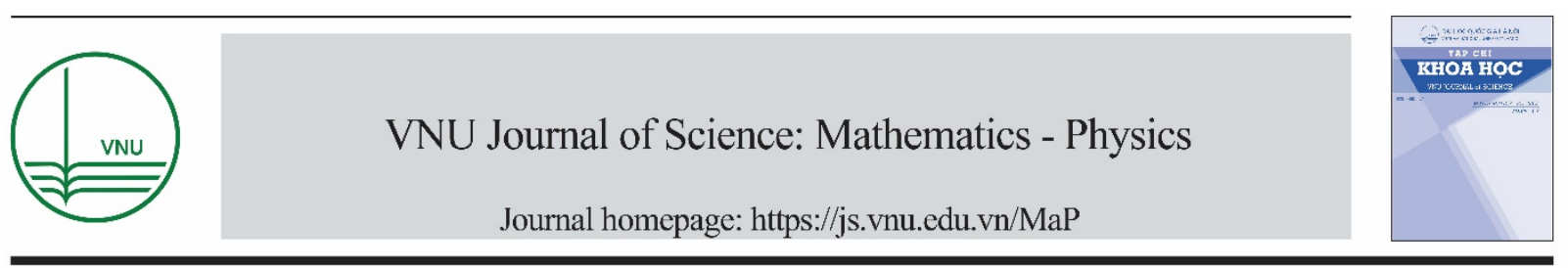

\title{
On the Stability Analysis of Delay Differential-Algebraic Equations
}

\author{
Ha Phi* \\ VNU University of Science, 334 Nguyen Trai, Thanh Xuan, Hanoi, Vietnam \\ Received 13 April 2018 \\ Revised 28 May 2018; Accepted 14 July 2018
}

\begin{abstract}
The stability analysis of linear time invariant delay differential- algebraic equations (DDAEs) is analyzed. Examples are delivered to demonstrate that the eigenvalue-based approach to analyze the exponential stability of dynamical systems is not valid for an arbitrarily high index system, and hence, a new concept of weak exponential stability (w.e.s) is proposed. Then, we characterize the w.e.s in term of a spectral condition for some special classes of DDAEs.

Keywords: Differential-algebraic equation, time delay, exponential stability, weak stability, simultaneous triangularizable.

Mathematics Subject Classification (2010): 34A09, 34A12, 65L05, 65H10.
\end{abstract}

\section{Introduction}

Our focus in the present paper is on the stability analysis of linear homogeneous, constant coefficients delay differential-algebraic equations (DDAEs) of the following form

$$
E \dot{x}(t)=A x(t)+B x(t-\tau), \text { for all } t \in[0, \infty),
$$

where $E, A, B \in \mathbb{R}^{n, n}, x:[-\tau, \infty) \rightarrow \mathbb{R}^{n}, \tau>0$ is a constant delay. DDAEs of the form (1) can be considered as a general combination of two important classes of dynamical systems, namely differentialalgebraic equations (DAEs)

$E \dot{x}(t)=A x(t)$, for all $t \in[0, \infty)$,

where the matrix $\mathrm{E}$ is allowed to be singular (det $E=0$ ), and delay-differential equations (DDEs)

$\dot{x}(t)=A x(t)+B x(t-\tau)$, for all $t \in[0, \infty)$,

\footnotetext{
* Tel.: 84-963304784.

Email: haphi.hus@vnu.edu.vn

https//doi.org/ 10.25073/2588-1124/vnumap.4264
} 
Due to the presence of both differential and difference operators, as well as the algebraic constraints, the study for DDAEs is much more complicated than that for standard DDEs or DAEs. The dynamics of DDAEs, therefore, as been strongly enriched, and many interesting properties, which occur neither for DAEs nor for DDEs, have been observed [1-4]. Due to these reasons, recently more and more attention has been devoted to DDAEs, [3-9]. One of the most important research topics in the qualitative theory of DDAE systems is the stability analysis, which has attracted many researches in recent years, [2, 5-7, 10]. It is well known, that for DDEs of the form (3), stability properties of the solution are closely related to spectral conditions of the matrix triple (I, A, B), see [11]. From the DAE side, not only the stability of (2) depends on spectral conditions of the matrix pencil $\lambda E-A$ but also the solvability is connected to the regularity of this pencil. Consequently, the stability of DDAEs are usually discussed under the regularity assumption of this pencil. Furthermore, one very important characteristic of DDAEs, namely index, has been underestimated in most of previous researches about the stability of DDAEs. The reason for this is due to two following facts: i) For DAE systems (without delay) of the form (2), an index does not affect the stability of the null solution. ii) Most of the considered DDAE systems, so far, are of index 1 , and also in this case, the stability is not influenced by an index. However, if an index of a DDAE is bigger than 1 then classical results on stability fail for DDAEs, see [2]. In fact, [2] is the only paper that the author aware of in the study of stability analysis for DDAE systems, whose indices are bigger than one. This paper aims to make some contribution to this research gap.

The short outline of this work is as follows. After some notations and auxiliary lemmas, in Section 2 we recall classical concept of (Lyapunov) exponential stability and its disadvantage, in order to motivate the weak exponential stability (w.e.s) concept. We also recall some important results about the stability and w.e.s for DDAE systems in some recent researches [2], [10]. Then, in Section 3 we extend the results in [10] for some bigger classes of DDAE systems. Finally, in Section 4 some conclusion and open questions are given.

In the following we denote by $\mathbb{N}\left(\mathbb{N}_{0}\right)$ the set of natural numbers (including 0 ), by $\mathbb{R}(\mathbb{C})$ the set of real (complex) numbers and $\mathbb{C}_{-}:=\{\lambda \in \mathbb{C} \mid \operatorname{Re}(\lambda)<0\}$. By $\|\cdot\|$ we denote a norm in $\mathbb{R}^{n}$, by $\mathbb{R}^{n, n}$ the set of real matrices of size $\mathrm{n}$ by $\mathrm{n}$ and by $I\left(I_{n}\right)$ the identity matrix (of size $\mathrm{n}$ by $\mathrm{n}$ ). As usual $x^{(\mathrm{j})}$ is the $\mathrm{j}$-th derivative of a function $x$. For $0 \leq p \leq \infty$, the set $C^{p}\left([-\tau, 0], \mathbb{R}^{n}\right)$ denotes the space of p-times continuously differentiable functions from $[-\tau, 0]$ to $\mathbb{R}^{n}$. These spaces are equipped with the norm defined by $\|\varphi\|_{C^{p}}:=\sum_{i=0}^{p} \sup _{t \in[-\tau, 0]}\left\|\varphi^{(\mathrm{i})}(\mathrm{t})\right\|$ to form a Banach space. For $\mathrm{p}=0$, we adopt the notation $C\left([-\tau, 0], \mathbb{R}^{n}\right)$ with the norm $\|\bullet\|_{\infty}:=\|\bullet\|_{C^{0}}$. Furthermore, let the set

$$
C_{b}^{\infty}\left([-\tau, 0], \mathbb{R}^{n}\right):=\left\{\varphi \in C^{\infty}\left([-\tau, 0], \mathbb{R}^{n}\right) \mid \sup _{i \geq 0} \sup _{t}\left\|\varphi^{(\mathrm{i})}(\mathrm{t})\right\|<\infty\right\}
$$

be equipped with the norm $\|\varphi\|_{C_{b}^{\infty}}:=\sup _{i \geq 0} \sup _{t \in[-\tau, 0]}\left\|\varphi^{(\mathrm{i})}(\mathrm{t})\right\|$ to form the Banach space. For a given and at most countable set $D \subset[0, \infty)$, by $C_{p w}^{p}\left([0, \infty), \mathbb{R}^{n}\right)$ we define the set of all p-times continuously differentiable at all except points belong to $\mathrm{D}$. For a function $x \in C_{p w}^{p}\left([0, \infty), \mathbb{R}^{n}\right)$, we adopt the notation

$$
\|x\|_{C_{p w}^{p}}:=\sum_{i=0}^{p}\left\|x^{(\mathrm{i})}(\mathrm{t})\right\| \text { for all } t \in[0, \infty) \backslash \mathrm{D} \text {. }
$$


To achieve uniqueness of solutions, analogous to the theory of DDEs, for DDAEs of the form (1) one typically has to prescribe an initial function, which takes the form

$$
\left.x\right|_{[-\tau, 0]}=\varphi:[-\tau, 0] \rightarrow \mathbb{R}^{n} .
$$

Within this paper, we use the concept of a piecewise differentiable solution, i.e. $x$ is continuous and $\mathrm{x}$ is continuously differentiable on $[0, \infty)$ except at the points belong to the set $D=\left\{\mathrm{i} \tau \mid \mathrm{i} \in \mathbb{N}_{0}\right\}$. Notice that, like DAEs, DDAEs are not solvable for arbitrary initial conditions, but they have to obey certain consistency conditions.

Definition 1 An initial function $\varphi$ is called consistent with (1) if the associated initial value problem (IVP) (1), (4) has at least one solution. System (1) is called solvable (resp. regular) if for every consistent initial function $\varphi$, the associated IVP (1), (4) has a solution (resp. has a unique solution).

Definition 2 Consider the DDAE (1). The matrix triple (E, A, B) is called regular if the two variable polynomial $P(\lambda, \omega)=\operatorname{det}(\lambda \mathrm{E}-\mathrm{A}-\omega \mathrm{B})$ is not identically zero. If, in addition, $B=0$ we say that the matrix pair $(E, A)$ (or the pencil $\lambda E-A)$ is regular. The sets

$$
\sigma(\mathrm{E}, \mathrm{A}, \mathrm{B}):=\left\{\lambda \in \mathbb{C} \mid \operatorname{det}\left(\lambda \mathrm{E}-\mathrm{A}-\mathrm{e}^{-\omega \tau} \mathrm{B}\right)=0\right\}, \rho(\mathrm{E}, \mathrm{A}, \mathrm{B}):=\mathbb{C} \backslash \sigma(\mathrm{E}, \mathrm{A}, \mathrm{B}),
$$

are called the spectrum and the resolvent set of (1), respectively.

In order to study DDAEs, strongly equivalent transformations are proposed as follows.

Definition 3 Two triples of matrices $\left(E_{1}, A_{1}, B_{1}\right)$ and $\left(E_{2}, A_{2}, B_{2}\right)$ in $\mathbb{C}^{m, n}$ are called strongly equivalent if there exist nonsingular matrices $S \in \mathbb{C}^{\mathrm{m}, \mathrm{m}}$ and $T \in \mathbb{C}^{\mathrm{n}, \mathrm{n}}$ such that $\left(E_{2}, A_{2}, B_{2}\right)=\left(\mathrm{SE}_{1} T, S_{1} T, S_{1} T\right)$. If this is the case, we write $\left(E_{2}, A_{2}, B_{2}\right) \sim\left(E_{1}, A_{1}, B_{1}\right)$.

Making use of strongly equivalent transformations, we can scale system (1) and change the variable as $x=T y$ to obtain a new system of the form

SET $\dot{y}(\mathrm{t})=S A T \mathrm{y}(\mathrm{t})+S B T \mathrm{y}(\mathrm{t}-\tau)$, for all $t \in[0, \infty)$.

We also note that the polynomial $P(\lambda, \omega)$, the spectrum $\sigma(\mathrm{E}, \mathrm{A}, \mathrm{B})$ and the resolvent $\rho(\mathrm{E}, \mathrm{A}, \mathrm{B})$ are preserved under strongly equivalent transformations. Furthermore, as shown in [10], the DDAE (1) is uniquely solvable only if the matrix triple $(\mathrm{E}, \mathrm{A}, \mathrm{B})$ is regular.

Lemma 1 (Kronecker-Weierstrass canonical form [12]) Consider the matrix pair (E, A) $\in\left(\mathbb{C}^{n, n}\right)^{2}$ and assume that it is regular. Then, there exist nonsingular matrices $\mathrm{S}, \mathrm{T}$ such that

$$
S E T=\left[\begin{array}{ll}
I & 0 \\
0 & N
\end{array}\right], S A T=\left[\begin{array}{ll}
J & 0 \\
0 & I
\end{array}\right],
$$

where $\mathrm{N}$ is nilpotent with the nilpoltency index $v(\mathrm{~N})$. Furthermore, one of the block row, and hence, the corresponding block column may not be present.

\section{Stability analysis of DDAEs}

In this section, we study the stability analysis of (1). As usual, we assume that the considered system is regular, i. e., for any consistent initial function $\varphi$, there exists a unique solution $\mathrm{x}(\mathrm{t})$. In comparison 
with DDEs, to introduce a new concept of exponential stability for the DDAE (1), the first and most natural idea would be adding a consistency assumption on an initial function $\varphi$, see e.g. [7]. We rephrase it in the next definition.

Definition 4 The null solution $\mathrm{x}=0$ of the DDAE (1) is called exponentially stable if there exist positive constants $\delta$ and $\gamma$ such that for any consistent initial function $\varphi \in C\left([-\tau, 0], \mathbb{R}^{n}\right)$, the solution $x=x(\mathrm{t}, \varphi)$ of the corresponding IVP to (1) satisfies

$$
\|x(\mathrm{t})\| \leq \delta e^{-\gamma t}\|\varphi\|_{\infty}, \text { for all } t \geq 0 \text {. }
$$

Notice that, for linear, homogeneous DDAEs, the exponential stability of the null solution and the one of an arbitrary solution are equivalent. Therefore, one can consider it as the exponential stability of the DDAE itself. For nonlinear systems, unfortunately, this does not hold true. Furthermore, one can directly see, that the stability of the DDAE (1) is preserved under strongly equivalent transformations. For the exponential stability of DDAEs, let us recall two important results presented in [11] and [2].

Proposition 1 ([11]) Consider a linear homogeneous DDE of the form

$$
\dot{x}(t)=A x(t)+B x(t-\tau) \text {, for all } t \in[0, \infty) \text {. }
$$

Then it is exponentially stable if and only if $\sigma(\mathrm{I}, \mathrm{A}, \mathrm{B}) \subset \mathbb{C}_{-}$.

Proposition 2 ([2]) If the DDAE (1) is strongly equivalent to the so-called strangeness-free formulation

$$
\begin{aligned}
& {\left[\begin{array}{c}
E_{1} \\
0
\end{array}\right] \dot{x}(\mathrm{t})=\left[\begin{array}{c}
A_{1} \\
0
\end{array}\right] x(\mathrm{t})+\left[\begin{array}{c}
B_{1} \\
0
\end{array}\right] x(\mathrm{t}-\tau), \text { for all } t \in[0, \infty),} \\
& \text { where }\left[\begin{array}{c}
E_{1} \\
A_{2}
\end{array}\right] \text { is nonsingular, then it is exponentially stable if and only if } \sigma(\mathrm{E}, \mathrm{A}, \mathrm{B}) \subset \mathbb{C}_{-} .
\end{aligned}
$$

Clearly, from the strangeness-free form (5), we see that $x(\mathrm{t})$ depends continuously on $x(\mathrm{t}-\tau)$. However, inherited from DAE theory, the solution $\mathrm{x}(\mathrm{t})$ usually depends not only on $x(\mathrm{t}-\tau)$ but also on its derivatives $\dot{x}(\mathrm{t}-\tau), \ldots, x^{(\mu)}(\mathrm{t}-\tau)$, for some $\mu \in \mathbb{N}$, which is called the strangeness-index of system (1). Therefore, Proposition 2 is no longer valid for general high-index DDAEs. This interesting effect has been observed in [2], as demonstrated in the following example.

Example 1 Consider the following DDAE on the time interval $I=[0, \infty)$.

$$
\left[\begin{array}{cccc}
1 & 0 & 0 & 0 \\
0 & 0 & 1 & 0 \\
0 & 0 & 0 & 1 \\
0 & 0 & 0 & 0
\end{array}\right]\left[\begin{array}{l}
\dot{x}_{1}(\mathrm{t}) \\
\dot{x}_{2}(\mathrm{t}) \\
\dot{x}_{3}(\mathrm{t}) \\
\dot{x}_{4}(\mathrm{t})
\end{array}\right]=\left[\begin{array}{cccc}
-1 & 0 & 0 & 0 \\
0 & 1 & 0 & 0 \\
0 & 0 & 1 & 0 \\
0 & 0 & 0 & 1
\end{array}\right]\left[\begin{array}{c}
x_{1}(\mathrm{t}) \\
x_{2}(\mathrm{t}) \\
x_{3}(\mathrm{t}) \\
x_{4}(\mathrm{t})
\end{array}\right]+\left[\begin{array}{cccc}
0 & 0 & 1 & 1 \\
0 & 0 & 0 & 0 \\
0 & 0 & 0 & 0 \\
1 & 0 & 0 & 0
\end{array}\right]\left[\begin{array}{c}
x_{1}(\mathrm{t}-\tau) \\
x_{2}(\mathrm{t}-\tau) \\
x_{3}(\mathrm{t}-\tau) \\
x_{4}(\mathrm{t}-\tau)
\end{array}\right]
$$

From the equations of system (6), we can directly obtain a new system

$$
\dot{x}_{1}(\mathrm{t})=-x_{1}(\mathrm{t})+\frac{1}{2}\left(x_{1}(\mathrm{t}-2 \tau)+\dot{x}_{1}(\mathrm{t}-2 \tau)\right)
$$




$$
\begin{gathered}
0=x_{2}(\mathrm{t})-\ddot{x}_{1}(\mathrm{t}-\tau) / 2, \\
0=x_{3}(\mathrm{t})-\dot{x}_{1}(\mathrm{t}-\tau) / 2, \\
0=x_{4}(\mathrm{t})-x_{1}(\mathrm{t}-\tau) / 2 .
\end{gathered}
$$

Clearly, equations (7b), (7c) imply that system (6) is unstable in the classical sense, since on the interval $[0, \tau]$ we have $x_{2}(\mathrm{t})=\ddot{\varphi}_{1}(\mathrm{t}-\tau) / 2$, and $x_{3}(\mathrm{t})=\dot{\varphi}_{1}(\mathrm{t}-\tau) / 2$. Consequently, system (6) is not exponentially stable. Nevertheless, one can directly verify that the spectrum $\sigma(\mathrm{E}, \mathrm{A}, \mathrm{B})$ is $\sigma(\mathrm{E}, \mathrm{A}, \mathrm{B})=\{-1\} \cup\{(\ln 2+2 \mathrm{k} \pi \mathrm{i}) / 2 \tau, \mathrm{k} \in \mathbb{Z}\} \subset \mathrm{C}_{-}$, which would suggest the completely wrong prediction.

Besides that, the existence of a continuous solution $x$ is only obtained when an initial function belongs to the space $C^{2}\left([-\tau, 0], \mathbb{R}^{n}\right)$ of two times continuously differentiable functions. If this is the case, the neutral DDE (7a) is exponentially stable whenever the set of initial function is restricted to the Banach space $\left(C^{1}\left([-\tau, 0], \mathbb{R}^{n}\right),\|\cdot\|_{C^{1}}\right)$. Furthermore, if the initial function $\varphi$ is in the class $C^{3}$, then the solution's component $x_{1}$ also belongs to the class $C^{3}$. Under this smoothness assumption, taking the second derivative of (7a) and making use of (7b), we obtain

$$
\dot{x}_{2}(\mathrm{t})+x_{2}(\mathrm{t})=\frac{1}{2}\left(x_{2}(\mathrm{t}-2 \tau)+\dot{x}_{2}(\mathrm{t}-2 \tau)\right) \text {, for all } \mathrm{t} \geq 0 .
$$

This equation also guarantees the exponential stability of the component $x_{2}$ as long as the initial condition $\left.x_{2}\right|_{[-\tau, 0]} \in C^{1}$, which clearly holds since $\varphi_{2} \in C^{3}$. Similarly, we have the exponential stability of the component $x_{3}$.

Example 1 have shown, that the spectral location will only give a right prediction to the behaviour of the solution when the initial function belongs to a suitable function space. Therefore, it raises two important questions. Firstly, for which type of DDAEs, the condition $\sigma(\mathrm{E}, \mathrm{A}, \mathrm{B}) \subset \mathbb{C}$ _still implies the exponential stability of the system. Secondly, for DDAEs of high-index, how to generalize the stability concept is such a way that systems like (6) are still (exponentially) stable. In the rest of this section we will partially answer these questions.

Definition 5 The homogeneous DDAE (1) is called non-advanced (or impulse-free) if for consistent initial function $\varphi \in C\left([-\tau, 0], \mathbb{R}^{n}\right)$, there exists a unique solution $\mathrm{x}$ to the IVP (1), (4).

The following lemma, taken from [4], gives a strangeness-free formulation for DDAEs.

Lemma 2 Consider the DDAE (1). Furthermore, assume that the IVP (1), (4) has a unique solution for every consistent initial function $\varphi \in C\left([-\tau, 0], \mathbb{R}^{n}\right)$. Moreover, assume that the DDAE (1) is nonadvanced. Then (1) can be transformed to the strangeness-free formulation (5).

Combining Proposition 2 and Lemma 2, we obtain the following result, which characterizes the exponential stability of the DDAE (1).

Proposition 3 Consider the linear, homogeneous DDAE (1). Then, (1) is exponentially stable if and only if the following assertions hold.

i) The DDAE (1) is non-advanced. 
ii) The spectrum $\sigma(\mathrm{E}, \mathrm{A}, \mathrm{B})$ lies on the open left half plane.

Now let us move to the second question mentioned above. Example 1 motivates a new concept of exponential stability for DDAE.

Definition 6 The null solution $x=0$ of the DDAE (1) is called $C^{p}$-weakly exponentially stable (w.e.s.) if there exist an integer $0 \leq p \leq \infty$ and positive constants $\delta$ and $\gamma$ such that for any consistent initial function $\varphi \in C^{p}\left([-\tau, 0], \mathbb{R}^{n}\right)$, the solution $x=x(\mathrm{t}, \varphi)$ of the corresponding IVP to (1) satisfies $\|x(\mathrm{t})\| \leq \delta e^{-\gamma t}\|\varphi\|_{C^{p}}$, for all $t \geq 0$.

Here $\gamma$ is called the decay rate of $\mathrm{x}(\mathrm{t})$. The minimum $p \in \mathbb{N}_{0}$ such that the DDAE (1) is $C^{p}$-w.e.s is called the $D$-perturbation index of the DDAE (1).

Notice that the (classical) exponential stability is exactly $C^{0}$-w.e.s.. Furthermore, even though $C^{p}$ -w.e.s. has been considered for ODEs and PDEs as well, till now we are not aware of any reference for DDAEs.

Remark 1 i) For any $p \leq q \in \mathbb{N}_{0}$ and any $\varphi \in C^{q}\left([-\tau, 0], \mathbb{R}^{n}\right)$, due to the estimation $\|\varphi\|_{C^{p}} \leq\|\varphi\|_{C^{q}}$

we see that if the null solution is $C^{p}$-w.e.s. then it is $C^{q}$-w.e.s.. We, however, notice that the space of consistent initial functions, while considering the norm $\|\cdot\|_{C^{q}}$, is strictly reduced, since $C^{q}\left([-\tau, 0], \mathbb{R}^{n}\right) \varsubsetneqq C^{p}\left([-\tau, 0], \mathbb{R}^{n}\right)$.

ii) The D-perturbation index proposed in Definition 6 is motivated from the concept of perturbation index of DAEs (without delay), which has been proposed and intensively studied, for details see [9] and the references therein. The relation between these indices will be the topic for future research.

Clearly, system (6) fits perfectly into this case, since the solution $\mathrm{x}(\mathrm{t})$ satisfies the estimation

$\|x\| \leq\|x\|_{C^{1}} \leq 2 e^{-t \ln 2 / \tau}\|\varphi\|_{C^{1}}$. However, except for the recent research [10], till now we have not found any reference on characterizations of $C^{p}$-w.e.s. systems. In the following propositions we recall two major results in [10].

Proposition 4 Suppose that $(\mathrm{E}, \mathrm{A}, \mathrm{B}) \in\left(\mathbb{C}^{n, n}\right)^{3}$ is a commutative triple, i.e., any two out of these three matrices commute. Then, there exists a nonsingular matrix $\mathrm{U}$ such that

$$
\begin{aligned}
& \left(\mathrm{UEU}^{-1}, \mathrm{UAU}^{-1}, \mathrm{UBU}^{-1}\right) \\
& =\left(\left[\begin{array}{cccc}
J^{E} & 0 & 0 & 0 \\
0 & N_{2}^{E} & 0 & 0 \\
0 & 0 & N_{3}^{E} & 0 \\
0 & 0 & 0 & N_{4}^{E}
\end{array}\right],\left[\begin{array}{cccc}
A_{1} & 0 & 0 & 0 \\
0 & J^{A} & 0 & 0 \\
0 & 0 & N_{3}^{A} & 0 \\
0 & 0 & 0 & N_{4}^{A}
\end{array}\right],\left[\begin{array}{cccc}
B_{1} & 0 & 0 & 0 \\
0 & B_{2} & 0 & 0 \\
0 & 0 & J^{B} & 0 \\
0 & 0 & 0 & N_{4}^{B}
\end{array}\right]\right)
\end{aligned}
$$

where $J^{E}, J^{A}, J^{B}$ are nonsingular, $N_{2}^{E}, N_{3}^{E}, N_{4}^{E}, N_{3}^{A}, N_{4}^{A}, N_{4}^{B}$ are nilpotent. Moreover, if the matrix triple $(\mathrm{E}, \mathrm{A}, \mathrm{B})$ is regular then the last block row and the last block column are not present.

Proposition 5 Assume that the DDAE (1) is regular. Moreover, suppose that the matrix triple (E, A, B) is commutative. Then the following assertions hold. 
i) The solution is exponentially stable if $\sigma(\mathrm{E}, \mathrm{A}, \mathrm{B}) \subset \mathbb{C}_{-}$, and the matrix $N_{2}^{E}$ in the equation (8) is identically 0 .

ii) The solution is $C^{\zeta-1}$-w.e.s. if $\sigma(\mathrm{E}, \mathrm{A}, \mathrm{B}) \subset \mathbb{C}_{-}$, where $\zeta$ is the nilpotency index of $N_{2}^{E}$, and $N_{2}^{E}$ is constructed as in (8). Consequently, the D-perturbation index of the DDAE (1) is at most $\zeta-1$.

\section{Stability analysis of DDAEs with non-commutative matrix coeficients}

Within this section we aim to study the weak exponential stability of a broader class of systems than those mentioned in Proposition 5. We will show that the null solution to (1) is still w.e.s whenever the spectrum satisfies the condition $\sigma(\mathrm{E}, \mathrm{A}, \mathrm{B}) \subset \mathbb{C}_{-}$, and the matrix coeficients are either weakly triangularizable or partially triangularizable. Let us begin with some definition.

Definition 7 a) The triple $(\mathrm{E}, \mathrm{A}, \mathrm{B}) \in\left(\mathbb{C}^{n, n}\right)^{3}$ are called simultaneously triangularizable if there exist a nonsingular matrix $\mathrm{S}$ such that $\mathrm{SES}^{-1}, S \mathrm{AS}^{-1}, S \mathrm{BS}^{-1}$ are upper triangular matrices.

b) The triple $(\mathrm{E}, \mathrm{A}, \mathrm{B}) \in\left(\mathbb{C}^{n, n}\right)^{3}$ are called weakly triangularizable if there exist nonsingular matrices $\mathrm{S}$, T such that $S E T, S A T, S B T$ are upper triangular matrices.

Simultaneously triangularizable matrices have been intensively studied, for details see the monograph by Radjavi and Rosenthal [14] and the references therein. This class of systems is much broader than the class of commutative matrices, see Chapter 1, [14]. However, until now there are not many results on weakly triangularizable matrices. The following lemma gives us a necessary and sscient condition for the weak- and simultaneous- triangularizability of three matrices E, A, B.

Lemma 3 Consider three matrices $(E, A, B) \in\left(\mathbb{C}^{n, n}\right)^{3}$ associated with the DDAE (1). Then, the triple $(\mathrm{E}, \mathrm{A}, \mathrm{B})$ is weakly triangularizable if and only if there exists a nonsingular matrix $\mathrm{X}$ such that all three matrices $A X B-B X A, A X C-C X A, B X C-C X B$ are nilpotent. Furthermore, if $X=I_{n}$ then E, A, B are simultaneously triangularizable.

Proof The second claim of this proposition is taken from Theorem 1.3.2 [14]. The proof of the first claim can be directly obtained by using the similar arguments and by taking $\mathrm{X}=\mathrm{TS}$, where the matrices $\mathrm{S}$ and $\mathrm{T}$ are mentioned in Definition 7.

Now without loss of generality we assume that the matrices E, A, B are already in the upper triangular form. Thus, system (1) becomes

$$
\left[\begin{array}{cccc}
E_{11} & * & \cdots & * \\
& E_{22} & \cdots & * \\
& & \ddots & \vdots \\
& & & E_{j j}
\end{array}\right] \dot{x}(\mathrm{t})=\left[\begin{array}{cccc}
A_{11} & * & \ldots & * \\
& A_{22} & \cdots & * \\
& & \ddots & \vdots \\
& & & A_{j j}
\end{array}\right] x(\mathrm{t})+\left[\begin{array}{cccc}
B_{11} & * & \ldots & * \\
& B_{22} & \ldots & * \\
& & \ddots & \vdots \\
& & & B_{j j}
\end{array}\right] x(\mathrm{t}-\tau),
$$

where the matrix $E_{i i}, i=1, \ldots, j$, are upper triangular, and for each of them, all of its elements on the main diagonal are simultaneously zero or nonzero. We notice that the sizes of three matrices in each triple $\left(E_{i i}, A_{i i}, B_{i i}\right)$ must be equal. Nevertheless, the sizes of different triples may be different. To analyze the stability of (9), by suitably scaling the system, in fact we only need to take care of four typical cases as follows. 


$$
\begin{aligned}
& \left(E_{i i}, A_{i i}, B_{i i}\right)=\left(\left[\begin{array}{ccc}
1 & \ldots & * \\
& \ddots & \vdots \\
& & 1
\end{array}\right],\left[\begin{array}{ccc}
* & \ldots & * \\
& \ddots & \vdots \\
& & *
\end{array}\right],\left[\begin{array}{ccc}
* & \ldots & * \\
& \ddots & \vdots \\
& & *
\end{array}\right]\right) \\
& \left(E_{i i}, A_{i i}, B_{i i}\right)=\left(\left[\begin{array}{ccc}
0 & \ldots & * \\
& \ddots & \vdots \\
& & 0
\end{array}\right],\left[\begin{array}{ccc}
1 & \ldots & * \\
& \ddots & \vdots \\
& & 1
\end{array}\right],\left[\begin{array}{ccc}
* & \ldots & * \\
& \ddots & \vdots \\
& & *
\end{array}\right]\right) \\
& \left(E_{i i}, A_{i i}, B_{i i}\right)=\left(\left[\begin{array}{ccc}
0 & \ldots & * \\
& \ddots & \vdots \\
& & 0
\end{array}\right],\left[\begin{array}{ccc}
0 & \ldots & * \\
& \ddots & \vdots \\
& & 0
\end{array}\right],\left[\begin{array}{ccc}
1 & \ldots & * \\
& \ddots & \vdots \\
& & 1
\end{array}\right]\right) \\
& \left(E_{i i}, A_{i i}, B_{i i}\right)=\left(\left[\begin{array}{ccc}
0 & \ldots & * \\
& \ddots & \vdots \\
& & 0
\end{array}\right],\left[\begin{array}{ccc}
0 & \ldots & * \\
& \ddots & \vdots \\
& & 0
\end{array}\right],\left[\begin{array}{ccc}
0 & \ldots & * \\
& \ddots & \vdots \\
& & 0
\end{array}\right]\right)
\end{aligned}
$$

Notice that blocks of the form (10d) could not occur in (9), due to the unique solvability of the DDAE (1). The following two lemmata will be very useful for our study later.

Lemma 4 Consider the corresponding IVP for the DDE

$$
\dot{x}(t)=A x(t)+B x(t-\tau)+\mathrm{f}(\mathrm{t}), \text { for all } t \in[0, \infty),
$$

and assume that the spectrum $\sigma(\mathrm{E}, \mathrm{A}, \mathrm{B}) \subset \mathbb{C}_{-}$and for some $p \in \mathbb{N}_{0}$, the initial function $\varphi \in C^{p}\left([-\tau, 0], \mathbb{R}^{n}\right)$ and $f \in C_{p w}^{p}\left([-\tau, 0], \mathbb{R}^{n}\right)$. Furthermore, assume that $f$ decays exponentially in the norm $\|\cdot\|_{C_{p w}^{p}}$, i. e. , $\|\mathrm{f}(\mathrm{t})\|_{C_{p w}^{p}} \leq C e^{-\gamma t}$, for all $t \in[0, \infty) \backslash \mathrm{D}$, where $C, \gamma>0$ are two positive constants. Then, $x \in C_{p w}^{p+1}\left([-\tau, 0], \mathbb{R}^{n}\right)$ and it also decays exponentially in the $\|\cdot\|_{C_{p w}^{p}}$-norm, i.e. $\|\mathrm{x}(\mathrm{t})\| \leq \tilde{C} e^{-\gamma t}\|\varphi\|_{\infty}$ for some constant $\tilde{C}$ and for all $t \in[0, \infty) \backslash \mathrm{D}$.

Proof To keep the brevity of this article, we will omit the detailed proof, which can be found in [15].

Lemma 5 Consider the corresponding IVP for the scalar difference equation

$0=x(t)+b x(t-\tau)+\mathrm{f}(\mathrm{t})$, for all $t \in[0, \infty)$.

Moreover, assume that $|b|<1$ and for some $p \in \mathbb{N}_{0}$, the initial function $\varphi \in C^{p}\left([-\tau, 0], \mathbb{R}^{n}\right)$ and $f \in C_{p w}^{p}\left([-\tau, 0], \mathbb{R}^{n}\right)$. Furthermore, assume that $f$ decays exponentially in the norm $\|\cdot\|_{C_{p w}^{p}}$, i. e. , $\|\mathrm{f}(\mathrm{t})\|_{C_{p w}^{p}} \leq C e^{-\gamma_{1} t}$, for all $t \in[0, \infty) \backslash \mathrm{D}$, where $C, \gamma_{1}>0$ are two positive constants.

Then, $\quad x \in C_{p w}^{p}\left([-\tau, 0], \mathbb{R}^{n}\right)$ and it also decays exponentially in the $\|\cdot\|_{C_{p w}^{p}}$-norm, i.e. $\|\mathrm{x}(\mathrm{t})\| \leq \tilde{C} e^{-\gamma t}\|\varphi\|_{\infty}$ for some positive constants $\tilde{C}, \gamma$ and for all $t \in[0, \infty) \backslash \mathrm{D}$. 
Proof Let $\gamma=\min \left\{\gamma_{1},-\frac{\ln |\mathrm{b}|}{\tau}\right\}$, we see that $\|\mathrm{f}(\mathrm{t})\|_{C_{p w}^{p}} \leq C e^{-\gamma t}$, for all $t \in[0, \infty) \backslash \mathrm{D}$.

By simple induction, we obtain the solution $x(t)$ as

$x(t)=\mathrm{b}^{[t / \tau]+1} \varphi(\mathrm{t}-[t / \tau] \tau-\tau)+\sum_{i=0}^{[t / \tau]} f(\mathrm{t}-\mathrm{i} \tau) b^{i}$, for all $t \in[0, \infty) \backslash \mathrm{D}$,

and hence, we have the following estimation for all $t \in[0, \infty) \backslash \mathrm{D}$.

$$
\begin{aligned}
& x(t) \leq|b|^{[t / \tau]+1}\|\varphi\|_{C^{p}}+\sum_{i=0}^{[t / \tau]} C e^{-\gamma(t-i \tau)}|b|^{i}, \\
& \leq|b|^{[t / \tau]+1}\|\varphi\|_{C^{p}}+\sum_{i=0}^{[t / \tau]} C e^{-\gamma t} e^{i(\gamma \tau+\ln \mid b)}, \\
& \leq|b|^{t / \tau}\|\varphi\|_{C^{p}}+\sum_{i=0}^{[t / \tau]} C e^{-\gamma t} e^{i(\gamma \tau+\ln |b|)}, \text { (since }|b|<1 \text { ), } \\
& \leq e^{-\gamma t}\|\varphi\|_{C^{p}}+C e^{-\gamma t} \frac{t}{1-e^{\gamma \tau+\ln |b|}},(\text { since } \gamma \tau+\ln |\mathrm{b}| \leq 0 \text { ), }
\end{aligned}
$$

which implies the $C^{p}$-exponential decay of the function $x$ for all $t \in[0, \infty) \backslash D$.

To illustrate our scheme to analyze the stability of the DDAE (1), we consider the following example, where all three cases (10a)-(10c) occur and they are of size 2 by 2 .

Example 2 Consider the following DDAE on the time interval $I=[0, \infty)$.

$\left[\begin{array}{cc:cc:cc}1 & e_{12} & e_{13} & e_{14} & e_{15} & e_{16} \\ & 1 & e_{23} & e_{24} & e_{25} & e_{26} \\ \hdashline & 0 & e_{34} & e_{35} & e_{36} \\ & & 0 & e_{45} & e_{45} \\ \hdashline & & & 0 & e_{56} \\ \hdashline & & & & 0\end{array}\right]\left[\begin{array}{c}\dot{x}_{1}(\mathrm{t}) \\ \dot{x}_{2}(\mathrm{t}) \\ \hdashline \dot{x}_{3}(\mathrm{t}) \\ \dot{x}_{4}(\mathrm{t}) \\ \hdashline \dot{x}_{5}(\mathrm{t}) \\ \dot{x}_{6}(\mathrm{t})\end{array}\right]=\left[\begin{array}{cc:cc:cc}a_{11} & a_{12} & a_{13} & a_{14} & a_{15} & a_{16} \\ & a_{22} & a_{23} & a_{24} & a_{25} & a_{26} \\ \hdashline & & 1 & a_{34} & a_{35} & a_{36} \\ & & & 1 & a_{45} & a_{45} \\ \hdashline & & & 0 & e_{56} \\ & & & & & 0\end{array}\right]\left[\begin{array}{c}x_{1}(\mathrm{t}) \\ x_{2}(\mathrm{t}) \\ \hdashline x_{3}(\mathrm{t}) \\ x_{4}(\mathrm{t}) \\ \hdashline x_{5}(\mathrm{t}) \\ x_{6}(\mathrm{t})\end{array}\right]$

$$
+\left[\begin{array}{cc:cc:cc}
b_{11} & b_{12} & b_{13} & b_{14} & b_{15} & b_{16} \\
& b_{22} & b_{23} & b_{24} & b_{25} & b_{26} \\
\hdashline & b_{33} & b_{34} & b_{35} & b_{36} \\
& & b_{44} & b_{45} & b_{45} \\
\hdashline & & & 1 & b_{56} \\
\hdashline & & & & 1
\end{array}\right]\left[\begin{array}{c}
x_{1}(\mathrm{t}-1) \\
x_{2}(\mathrm{t}-1) \\
\hdashline x_{3}(\mathrm{t}-1) \\
x_{4}(\mathrm{t}-1) \\
\hdashline x_{5}(\mathrm{t}-1) \\
x_{6}(\mathrm{t}-1)
\end{array}\right] .
$$

We will prove that if the spectrum $\sigma(\mathrm{E}, \mathrm{A}, \mathrm{B}) \subset \mathbb{C}_{-}$, then system (13) is w.e.s.

First we rewrite system (13) as follows. 


$$
\begin{aligned}
& {\left[\begin{array}{cc}
1 & e_{12} \\
& 1
\end{array}\right]\left[\begin{array}{l}
\dot{x}_{1}(\mathrm{t}) \\
\dot{x}_{2}(\mathrm{t})
\end{array}\right]=\left[\begin{array}{cc}
a_{11} & a_{12} \\
& a_{22}
\end{array}\right]\left[\begin{array}{l}
x_{1}(\mathrm{t}) \\
x_{2}(\mathrm{t})
\end{array}\right]+\left[\begin{array}{ll}
b_{11} & b_{12} \\
& b_{22}
\end{array}\right]\left[\begin{array}{l}
x_{1}(\mathrm{t}-1) \\
x_{2}(\mathrm{t}-1)
\end{array}\right]+\left[\begin{array}{l}
f_{1} \\
f_{2}
\end{array}\right],} \\
& {\left[\begin{array}{cc}
0 & e_{34} \\
& 0
\end{array}\right]\left[\begin{array}{c}
\dot{x}_{1}(\mathrm{t}) \\
\dot{x}_{2}(\mathrm{t})
\end{array}\right]=\left[\begin{array}{cc}
1 & a_{34} \\
& 1
\end{array}\right]\left[\begin{array}{l}
x_{1}(\mathrm{t}) \\
x_{2}(\mathrm{t})
\end{array}\right]+\left[\begin{array}{cc}
b_{33} & b_{34} \\
& b_{44}
\end{array}\right]\left[\begin{array}{l}
x_{1}(\mathrm{t}-1) \\
x_{2}(\mathrm{t}-1)
\end{array}\right]+\left[\begin{array}{c}
f_{3} \\
f_{4}
\end{array}\right],} \\
& {\left[\begin{array}{cc}
0 & e_{56} \\
& 0
\end{array}\right]\left[\begin{array}{c}
\dot{x}_{1}(\mathrm{t}) \\
\dot{x}_{2}(\mathrm{t})
\end{array}\right]=\left[\begin{array}{cc}
0 & a_{56} \\
& 0
\end{array}\right]\left[\begin{array}{l}
x_{1}(\mathrm{t}) \\
x_{2}(\mathrm{t})
\end{array}\right]+\left[\begin{array}{cc}
1 & b_{56} \\
& 1
\end{array}\right]\left[\begin{array}{l}
x_{1}(\mathrm{t}-1) \\
x_{2}(\mathrm{t}-1)
\end{array}\right]+\left[\begin{array}{c}
f_{5} \\
f_{6}
\end{array}\right],}
\end{aligned}
$$

where the inhomogeneities $f_{i}, i=1, \ldots, 6$, are multi-linear functions satisfies the following dependencies

$$
\begin{aligned}
& f_{1}=f_{1}\left(\dot{\mathrm{x}}_{3}(\mathrm{t}), \ldots, \dot{\mathrm{x}}_{6}(\mathrm{t}), \mathrm{x}_{3}(\mathrm{t}), \ldots, \mathrm{x}_{6}(\mathrm{t}), \mathrm{x}_{3}(\mathrm{t}-1), \ldots, \mathrm{x}_{6}(\mathrm{t}-1)\right), \\
& f_{2}=f_{2}\left(\dot{\mathrm{x}}_{3}(\mathrm{t}), \ldots, \dot{\mathrm{x}}_{6}(\mathrm{t}), \mathrm{x}_{3}(\mathrm{t}), \ldots, \mathrm{x}_{6}(\mathrm{t}), \mathrm{x}_{3}(\mathrm{t}-1), \ldots, \mathrm{x}_{6}(\mathrm{t}-1)\right), \\
& f_{3}=f_{3}\left(\dot{\mathrm{x}}_{5}(\mathrm{t}), \dot{\mathrm{x}}_{6}(\mathrm{t}), \mathrm{x}_{5}(\mathrm{t}), \mathrm{x}_{6}(\mathrm{t}), \mathrm{x}_{5}(\mathrm{t}-1), \mathrm{x}_{6}(\mathrm{t}-1)\right), \\
& f_{4}=f_{4}\left(\dot{\mathrm{x}}_{5}(\mathrm{t}), \dot{\mathrm{x}}_{6}(\mathrm{t}), \mathrm{x}_{5}(\mathrm{t}), \mathrm{x}_{6}(\mathrm{t}), \mathrm{x}_{5}(\mathrm{t}-1), \mathrm{x}_{6}(\mathrm{t}-1)\right), \\
& f_{5}=f_{6}=0 .
\end{aligned}
$$

Let us partition the initial function correspondingly, as $\varphi=\left[\begin{array}{llllll}\varphi_{1}^{T} & \varphi_{2}^{T} & \varphi_{3}^{T} & \varphi_{4}^{T} & \varphi_{5}^{T} & \varphi_{6}^{T}\end{array}\right]^{T}$.

Even though in this example $f_{5}=f_{6}=0$, in general, where more than three block equations are present, they would be multi-linear functions in the space $C_{p w}^{p}\left([-\tau, 0], \mathbb{R}^{n}\right)$ for some $p \in \mathbb{N}_{0}$, and they satisfy the exponential decay estimation for some positive constants $C, \gamma_{1}$, i. e.,

$$
\|\mathrm{f}(\mathrm{t})\|_{C_{p w}^{p}} \leq C e^{-\gamma_{1} t}, \text { for all } t \in[0, \infty) \backslash \mathrm{D} \text {. }
$$

Thus, due to Lemma 5, we have that $\left\|\mathrm{x}_{6}(\mathrm{t})\right\|_{C_{p w}^{p}} \leq C_{6} e^{-\gamma(t+1)}\left\|\varphi_{6}\right\|_{C^{p}}$, for all $t \in[0, \infty) \backslash \mathrm{D}$.

From the first equation of (14c), we have that $0=\mathrm{x}_{5}(\mathrm{t}-1)+\left(f_{5}-e_{56} \dot{\mathrm{x}}_{6}(\mathrm{t})+a_{56} \mathrm{x}_{6}(\mathrm{t})+\mathrm{b}_{56} \mathrm{x}_{6}(\mathrm{t}-1)\right)$, for all $t \in[0, \infty) \backslash \mathrm{D}$.

Due to the trivial observation that $x_{6}$ decays exponentially in the norm $\|\cdot\|_{C_{p w}^{p}}$ follows that $\dot{x}_{6}$ also decays exponentially, but in the norm $\|\cdot\|_{C_{p w}^{p-1}}$, we see that there exists a constant $C_{5}$ such that $\left\|\mathrm{x}_{5}(\mathrm{t})\right\|_{C_{p w}^{p-1}} \leq C_{5} e^{-\gamma t}\left\|\varphi_{5}\right\|_{C^{p-1}}$, for all $t \in[0, \infty) \backslash \mathrm{D}$.

Consequently, due to their definition, both $f_{3}$ and $f_{4}$ decay exponentially with the same rate in the $\|\cdot\|_{C_{p w}^{p-2}}$-norm. Now we proceed consecutively with two equations of (14b), then Lemma 5 follows that $x_{3}\left(\right.$ resp. $\left.x_{4}\right)$ decays exponentially in the $\|\cdot\|_{C_{p w}^{p-2}}$-norm (resp. $\|\cdot\|_{C_{p w}^{p-3}}$-norm). Finally, applying 
Lemma 4, we see that the vector-valued function $\left[\begin{array}{l}x_{1}(\mathrm{t}) \\ x_{2}(\mathrm{t})\end{array}\right]$ also decays exponentially in the $\|\cdot\|_{C_{p w}^{p-3}-}$ norm. Due to Remark 1 i), we see that all the components of $x(t)$ decays exponentially in the $\|\cdot\|_{C_{p w}^{p-3}}$ norm, and hence, $\mathrm{x}(\mathrm{t})$ is $C^{p}$-w.e.s. for any $p \geq 3$. Consequently, in general, the D-perturbation index of (13) is at most 3.

Using the same argument, we obtain the following theorem, which completely characterize the w.e.s to DDAE systems with weakly triangularizable matrix coeficients.

Theorem 1 Assume that the DDAE (1) is regular. Moreover, suppose that the matrix triple (E, A, B) is weakly triangularizable. Then, (1) is $C^{n-1}$-w.e.s. if $\sigma(\mathrm{E}, \mathrm{A}, \mathrm{B}) \subset \mathbb{C}_{-}$. The D-perturbation index of (1), therefore, is at most $n-1$.

Proof The proof is obtained by considering consecutively the scalar equations from the bottom up to the top and making use of Lemmata 4, 5 as in Example 2. We will omit the details here for the brevity of this work.

To illustrate our result let us consider Example 3.6 in [2].

Example 3 Consider the following DDAE on the time interval $I=[0, \infty)$.

$$
\left[\begin{array}{cccc}
1 & 0 & 0 & 0 \\
0 & 0 & 1 & 0 \\
0 & 0 & 0 & 1 \\
0 & 0 & 0 & 0
\end{array}\right]\left[\begin{array}{c}
\dot{x}_{1}(\mathrm{t}) \\
\dot{x}_{2}(\mathrm{t}) \\
\dot{x}_{3}(\mathrm{t}) \\
\dot{x}_{4}(\mathrm{t})
\end{array}\right]=\left[\begin{array}{cccc}
-1 & 0 & 0 & 0 \\
0 & 2 & 0 & 0 \\
0 & 0 & 2 & 0 \\
0 & 0 & 0 & 2
\end{array}\right]\left[\begin{array}{c}
x_{1}(\mathrm{t}) \\
x_{2}(\mathrm{t}) \\
x_{3}(\mathrm{t}) \\
x_{4}(\mathrm{t})
\end{array}\right]+\left[\begin{array}{cccc}
0 & 0 & 0 & 1 \\
0 & 1 & 0 & 0 \\
0 & 0 & 1 & 0 \\
0 & 0 & 0 & 1
\end{array}\right]\left[\begin{array}{c}
x_{1}(\mathrm{t}-\tau) \\
x_{2}(\mathrm{t}-\tau) \\
x_{3}(\mathrm{t}-\tau) \\
x_{4}(\mathrm{t}-\tau)
\end{array}\right] .
$$

We can directly compute the spectrum, which is

$$
\sigma(\mathrm{E}, \mathrm{A}, \mathrm{B})=\{-1\} \cup\{(\ln 2+2 \mathrm{k} \pi \mathrm{i}) / 2 \tau, \mathrm{k} \in \mathbb{Z}\} \subset \mathrm{C}_{-} \text {, }
$$

which lies entirely on the open left half plane. As observed in [4], this DDAE is unstable in the classical sense. However, since all matrix coeficients are already in the upper triangular form, Theorem 1 implies that this DDAE is $C^{3}$-w.e.s.

Remark 2 It should be noted that, Theorem 1 only gives the upper bound for the D-perturbation index of (1). For example, the DDAE (13) has D-perturbation index $\leq 3$, even though its size is 6 by 6 .

In many applications, for example $[2,7,8]$, the matrix pair $(E, A)$ is regular. This fact suggests us to make use of the Kronecker-Weierstrass canonical form in Lemma 1 to the matrix pair (E,A) and then, to use strongly equivalent transformations in order to bring the DDAE (1) into the following form

$$
\left[\begin{array}{cc}
I & 0 \\
0 & N
\end{array}\right]\left[\begin{array}{c}
\dot{x}_{1}(\mathrm{t}) \\
\dot{x}_{2}(\mathrm{t})
\end{array}\right]=\left[\begin{array}{cc}
J & 0 \\
0 & I
\end{array}\right]\left[\begin{array}{c}
x_{1}(\mathrm{t}) \\
X_{2}(\mathrm{t})
\end{array}\right]+\left[\begin{array}{cc}
B_{1} & B_{2} \\
B_{3} & B_{4}
\end{array}\right]\left[\begin{array}{c}
x_{1}(\mathrm{t}-\tau) \\
x_{2}(\mathrm{t}-\tau)
\end{array}\right] \text {. }
$$

Here we notice that the matrix $\mathrm{N}$ is nilpotent with the nilpoltency index $v(\mathrm{~N})$. We propose the concept of partial triangularizability in the next definition.

Definition 8 Consider the DDAE (1) and assume that the matrix pair (E, A) is regular, so that one can bring (1) to the form (16) using strongly equivalent transformation. The triple (E, A, B) is called partially triangularizable if the following conditions hold. 
i) The identity $B_{3}=0$ holds true.

ii) The matrices $N, B_{4}$ are simultaneously triangularizable.

Notice that the triple (E, A, B) is partial triangularizable if and only if $B_{3}=0$ and the matrix triple $\left(\mathrm{N}, I, \mathrm{~B}_{4}\right)$ is simultaneously triangularizable. Thus, Theorem 1 can be applied to the second block row equation of (16), which leads us to the following corollary.

Corollary 1 Consider the DDAE (1) and assume that the matrix pair (E, A) is regular, so that one can bring (1) to the form (16) using strongly equivalent transformations. Furthermore, assume that the triple (E, A, B) is partially triangularizable. If $\sigma(\mathrm{E}, \mathrm{A}, \mathrm{B}) \subset \mathbb{C}_{-}$, then $(1)$ is $C^{\nu(\mathrm{N})-1}$-w.e.s..

Proof First we apply Theorem 1 to the second block row equation of (16), which is

$N \dot{x}_{2}(\mathrm{t})=x_{2}(\mathrm{t})+\mathrm{B}_{4} x(\mathrm{t}-\tau)$.

Thus, $x_{2}$ is $C^{v(\mathrm{~N})-1}$-w.e.s. Then Lemma 4 applied to the DDE

$\dot{x}_{1}(\mathrm{t})=J X_{1}(\mathrm{t})+\mathrm{B}_{1} x_{1}(\mathrm{t}-\tau)+\mathrm{B}_{2} x_{2}(\mathrm{t}-\tau)$

gives us the desire result.

To illustrate this result let us consider again Example 3.6 in [2].

Example 4 Consider the following DDAE on the time interval $I=[0, \infty)$.

$$
\left[\begin{array}{cccc}
1 & 0 & 0 & 0 \\
0 & 0 & 1 & 0 \\
0 & 0 & 0 & 1 \\
0 & 0 & 0 & 0
\end{array}\right]\left[\begin{array}{c}
\dot{x}_{1}(\mathrm{t}) \\
\dot{x}_{2}(\mathrm{t}) \\
\dot{x}_{3}(\mathrm{t}) \\
\dot{x}_{4}(\mathrm{t})
\end{array}\right]=\left[\begin{array}{cccc}
-1 & 0 & 0 & 0 \\
0 & 2 & 0 & 0 \\
0 & 0 & 2 & 0 \\
0 & 0 & 0 & 2
\end{array}\right]\left[\begin{array}{c}
x_{1}(\mathrm{t}) \\
x_{2}(\mathrm{t}) \\
x_{3}(\mathrm{t}) \\
x_{4}(\mathrm{t})
\end{array}\right]+\left[\begin{array}{cccc}
0 & 0 & 0 & 1 \\
0 & 1 & 0 & 0 \\
0 & 0 & 1 & 0 \\
0 & 0 & 0 & 1
\end{array}\right]\left[\begin{array}{c}
x_{1}(\mathrm{t}-\tau) \\
x_{2}(\mathrm{t}-\tau) \\
x_{3}(\mathrm{t}-\tau) \\
x_{4}(\mathrm{t}-\tau)
\end{array}\right] .
$$

Corollary 1 applied to this DDAE turns out that this DDAE is $C^{2}$-w.e.s.. Thus, the D-perturbation index of (17) is upper bounded by 2 . This is a better bound than the one provided by Theorem 1 , while Proposition 5 could not be applied, since three matrix coeficients in system (17) do not commute.

\section{Conclusion and outlooks}

In this paper, we have extended the recent stability result in [10] to a broader class of linear delay differential-algebraic equations (DDAEs). Weak exponential stability is characterized in term of spectral conditions for systems whose matrix coeficients are weakly-, simultaneously- or partially triangularizable. We notice that the Kronecker-Weierstrass canonical form, up to now, is not stably computed, and hence, another decompositions for matrix pairs, for example, QZ decomposition, could be used to generalize the concept of partial triangularizability. The second research direction is to investigate the Lambert function for DDAEs, which has only been done for DDEs. Furthermore, it has been shown in [16] that some important spectral/stability results for Lambert function are only valid when the simultaneous triangularizability of the system coeficients is assumed. Therefore, studying the relation between the Lambert function and the w.e.s of DDAEs would be an interesting topic. Finally, further investigation on the relation between the D-perturbation index and the perturbation index, and sharper estimations for the D-perturbation index will be the third research interest in the future. 


\section{Acknowledgements}

The author would like to thank an anonymous referee for his suggestions that strongly improve the quality of this paper. This research is funded by the VNU University of Science under the project number TN.17.01

\section{References}

[1] S. L. Campbell. Nonregular 2D descriptor delay systems. IMA J. Math. Control Appl., 12:57-67, 1995.

[2] Nguyen Huu Du, Vu Hoang Linh, Volker Mehrmann, and Do Duc Thuan. Stability and robust stability of linear time-invariant delay differential- algebraic equations. SIAM J. Matr. Anal. Appl., 34(4):1631-1654, 2013.

[3] Phi Ha and Volker Mehrmann. Analysis and reformulation of linear delay differential-algebraic equations. Electr. J. Lin. Alg., 23:703-730, 2012.

[4] Phi Ha and Volker Mehrmann. Analysis and numerical solution of linear delay differential-algebraic equations. BIT, 56:633 - 657, 2016.

[5] S. L. Campbell and V. H. Linh. Stability criteria for differential-algebraic equations with multiple delays and their numerical solutions. Appl. Math Comput., 208(2):397 - 415, 2009.

[6] Emilia Fridman. Stability of linear descriptor systems with delay: a Lyapunov-based approach. J. Math. Anal. Appl., 273(1):24 - 44, 2002.

[7] W. Michiels. Spectrum-based stability analysis and stabilisation of systems described by delay differential algebraic equations. IET Control Theory Appl., 5(16):1829-1842, 2011.

[8] L. F. Shampine and P. Gahinet. Delay-differential-algebraic equations in control theory. Appl. Numer. Math., 56(34):574-588, March 2006.

[9] H. Tian, Q. Yu, and J. Kuang. Asymptotic stability of linear neutral delay differential-algebraic equations and Runge-Kutta methods. SIAM J. Numer. Anal., 52(1):68-82, 2014.

[10] Phi Ha. Spectral characterizations of solvability and stability for delay differential-algebraic equations. Accepted for Acta Math. Vietnamica, url: https://arxiv.org/abs/1802.01148, 2018.

[11] J.K. Hale and S.M.V. Lunel. Introduction to Functional Differential Equations. Springer, 1993.

[12] P. Kunkel and V. Mehrmann. Differential-Algebraic Equations - Analysis and Numerical Solution. EMS Publishing House, Zürich, Switzerland, 2006.

[13] E. Hairer, C. Lubich, and M. Roche. The Numerical Solution of Differential-Algebraic Systems by Runge-Kutta Methods. Springer-Verlag, Berlin, Germany, 1989.

[14] H. Radjavi and P. Rosenthal. Simultaneous Trianqularizability. Universitext, Springer, New york, 2000.

[15] Richard Bellman and Kenneth L. Cooke. Differential-difference equations. Mathematics in Science and Engineering. Elsevier Science, 1963.

[16] E. Jarlebring. The Lambert $\mathrm{W}$ function and the spectrum of some multi- dimensional time-delay systems. Automatica, 43(12):2124 - 2128, 2007. 